\title{
Mixture Designs Generated by Orthogonal Arrays Developed Using Difference Schemes
}

\author{
Poonam Singh \\ Department of Statistics, \\ University of Delhi, Delhi, 110007, India. \\ E-mail: psingh@stats.du.ac.in \\ Vandana Sarin \\ Department of Statistics, Kirori Mal College, \\ University of Delhi, Delhi, 110007, India. \\ E-mail: vandana_s_walia@kmc.du.ac.in \\ Neha Midha \\ Department of Statistics, \\ University of Delhi, Delhi, 110007, India. \\ Corresponding author: nehamidha1189@gmail.com
}

(Received May 6, 2020; Accepted July 29, 2020)

\begin{abstract}
This paper presents an algorithm for constructing mixture designs based on orthogonal arrays developed using difference schemes. The algorithm can also be applied to constrained mixture experiments. The algorithm allows the generation of efficient mixture designs for Scheffé canonical polynomials.
\end{abstract}

Keywords- Mixture experiments, Difference schemes, Restricted region, G-efficiency.

\section{Introduction}

In experiments with mixtures, the response is a function only of the proportions of the $q$ components present in the mixture and not of the total amount of the mixture. If $x_{i}$ is the proportion of $i^{\text {th }}$ component, $i=1,2, \ldots, q$, then

$0 \leq x_{i} \leq 1, \sum_{i=1}^{q} x_{i}=1$.

These restrictions force the factor space, of the $q$ components to take form of a $(q-1)$ dimensional simplex. The general purpose of mixture experimentation is to estimate the properties of an entire multicomponent system from only a limited number of observations. These observations are taken at preselected combinations of the components to determine which of the combinations in some sense optimize the response.

In many practical situations, one can encounter certain additional constraints that are placed on some or all component proportions besides (1). These are of the form

$0 \leq L_{i} \leq x_{i} \leq U_{i} \leq 1 ; i=1,2, \ldots, q$. 
International Journal of Mathematical, Engineering and Management Sciences

Vol. 5, No. 6, 1379-1391, 2020

https://doi.org/10.33889/IJMEMS.2020.5.6.102

where, $L_{i}$ and $U_{i}$ denote the lower bound and upper bound for the component proportion $x_{i} ; i=$ $1,2, \ldots, q$. These supplementary restrictions limit the experimentation to some sub-region of the simplex, thereby altering the shape of the experimental region from a simplex to an irregularly shaped convex polyhedron inside the simplex. In such situations, directing the design and modelling only to the sub-region can help in lowering the experimentation cost and time and increasing the precision of model estimates.

Canonical polynomials (Scheffé, 1958; Scheffé, 1963) are most widely used for fitting of mixture models to the data and are stated below:

Linear model: $Y=\sum_{i=1}^{q} \beta_{i} x_{i}+\varepsilon$

Quadratic model: $Y=\sum_{i=1}^{q} \beta_{i} x_{i}+\sum \sum_{i<j}^{q} \beta_{i j} x_{i} x_{j}+\varepsilon$.

Special cubic model: $Y=\sum_{i=1}^{q} \beta_{i} x_{i}+\sum \sum_{i<j}^{q} \beta_{i j} x_{i} x_{j}+\sum \sum \sum_{i<j<k}^{q} \beta_{i j k} x_{i} x_{j} x_{k}+\varepsilon$.

Restricted region has been explored in different ways. Extreme vertices designs (McLean and Anderson, 1966) were developed to satisfy both the constraints (1) and (2). A partial solution to the restricted exploration problem was provided by identifying the point of maximum interest and considering an ellipsoidal region around it (Thompson and Myers, 1968). Subsets of the extreme vertices were shown to provide precise estimates of the parameters of a linear model (Snee and Marquardt, 1974). An improved algorithm for mixture designs with components bounded by upper and lower bounds was established (Nigam et al., 1983). Computers were used to develop designs in constrained mixture spaces for the quadratic model (Snee, 1975). The restricted mixture region was investigated using symmetric-simplex design (Saxena and Nigam, 1977). A method of construction of mixture designs for the examination of the restricted region using factorials was deliberated (Murthy and Murty, 1983). The construction of mixture designs using Taguchi's fixed element orthogonal arrays was discussed (Singh et al., 2018).

Difference schemes were defined as a tool for constructing orthogonal arrays of strength two (Bose and Bush, 1952). An example of a difference scheme of index two based on the additive group of Galois Field was stated (Bose and Bush, 1952). The construction of difference scheme of index two, $D(2 p, 2 p, p)$, where $p$ is a prime or a power of a prime was studied (Masuyama, 1969; Jungnickel, 1979; Xu, 1979; Xiang, 1983). The methods of recursive construction of orthogonal arrays based on a series of difference schemes were specified (Shrikhande, 1964; Mukhopadhyay, 1981).

In this paper, we present an algorithm for constructing mixture designs based on orthogonal arrays generated using difference schemes. We have examined and compared the designs constructed through this algorithm with the existing designs based on G-efficiency. The manageable number of design points help in reducing the cost and time in statistical experiments.

\section{Orthogonal Arrays Based on Difference Schemes}

Hypercubus of strength ' $d$ ' were defined (Rao, 1946). Later, the definition of hypercube of strength $d$ was extended to cover a wider class of arrays called orthogonal arrays (Rao, 1947). An $N \times k$ array $A$ with entries from $S$ is said to be an orthogonal array $O A(N, k, s, t)$ with $s$ levels, strength $t$ and index $\lambda$ (for some $t$ in the range $0 \leq t \leq k$ ) if every $N \times t$ sub-array of $A$ contains each $t$-tuple 
International Journal of Mathematical, Engineering and Management Sciences

Vol. 5, No. 6, 1379-1391, 2020

https://doi.org/10.33889/IJMEMS.2020.5.6.102

based on $S$ exactly $\lambda$ times as a row. If $\lambda=1$, then such arrays are referred to as orthogonal arrays of index unity (Bush, 1952). Orthogonal arrays can be constructed using difference schemes.

An $r \times q$ array $D$ with the entries from a finite abelian group, $\mathcal{A}$ is called a difference scheme based on binary operation + , represented by $(\mathcal{A},+)$, if it has the property that for all $i$ and $j$ with $1 \leq$ $i, j \leq q, i \neq j$, the vector difference between the $i$ th and $j$ th columns contains every element of $\mathcal{A}$ equally often.

\subsection{Design Criteria}

If $X$ denotes the $N \times k$ design matrix, then a useful criterion for evaluating the design is the minimum-maximum variance criterion. This refers to minimizing the maximum variance of prediction over the experimental region, where the prediction variance at the point $x(1 \times k$ row vector) is given by $\sigma^{2} v$ and $v=x\left(X^{\prime} X\right)^{-1} x^{\prime}$. Computation of the maximum variance provides a criterion of how close is a design to optimality. The G-efficiency or Global efficiency of the design is given by,

$G-$ efficiency $($ percent of optimum $)=100 \mathrm{p} / \mathrm{Nd}$.

where, $p$ is the number of model parameters, $N$ is the number of design points and $d$ is the maximum value of $v$ over the experimental region.

As a practical rule of thumb, any design with a $G-$ efficiency $\geq 50 \%$ could be called good for practical purposes (Wheeler, 1972).

\section{OABMD Algorithm}

Let $D$ be a difference scheme based on $(\mathcal{A},+)$, where $\mathcal{A}=\left\{\sigma_{0}, \sigma_{1}, \ldots, \sigma_{s-1}\right\}$. Let $D_{i}$ denote the array obtained from $D$ by adding $\sigma_{i}$ to each of its entries. Juxtaposing the elements of $D_{i}$ 's develops into an orthogonal array of strength two. If $D$ is a difference scheme $D(r, q, s)$, then $A^{T}=$ $\left[\begin{array}{llll}D_{0} & D_{1} & \ldots & D_{s-1}\end{array}\right]$ is an $O A(r s, q, s, 2)$. Appending a symbol to each run of $D_{i}$ gives another orthogonal array $O A(r s, q+1, s, 2)$.

We now present Orthogonal Array Based Mixture Design (OABMD) algorithm for constructing $q$ component mixture designs using orthogonal arrays based on difference schemes.

Step 1: Construct an orthogonal array $O A(r s, q, s, 2)$ using the difference scheme $D(r, q, s)$. Denote this matrix by $A$.

Step 2: Create a matrix $M$ of order $q \times q$ which is symmetrical but not orthogonal, having all elements as integers with sum of elements in each row and each column being zero. The choice of $M$ is arbitrary and is useful in getting more vertices of the experimental region in the design (Murthy and Murty, 1983).

Step 3: Identify the minimum value in each column of $A \times M$ and subtract it from all the entries of that corresponding column to create a new matrix $T$. 
International Journal of Mathematical, Engineering and Management Sciences

Vol. 5, No. 6, 1379-1391, 2020

https://doi.org/10.33889/IJMEMS.2020.5.6.102

Step 4: Obtain the row totals for matrix $T$. Divide the entries of each row of $T$ by its corresponding row total to obtain a new matrix $Z$. The resultant matrix is a mixture design satisfying $0 \leq z_{i} \leq 1$ and $\sum_{i=1}^{q} z_{i}=1, z_{i}$ being the proportion for $i^{t h}$ component.

Further, if the mixture experiment has to be performed in the restricted region, where each component is bounded by lower or upper bounds, or both, then proceed as follows:

Step 5: Rank the components in order of their increasing ranges $R_{i}=\left(U_{i}-L_{i}\right)$ such that $X_{1}$ has the smallest range and $X_{q}$ has the largest range, assuming range to be inversely proportional to the importance (in terms of cost, effectiveness, etc.) of the components in the experiment.

Step 6: Using the transformation (Saxena and Nigam, 1977), $X_{i}=L_{i}+\left(R_{i} \times z_{i}\right)$, compute the entries for the first $(q-1)$ components of the design matrix $X$. The levels of $X_{q}$ are obtained by $X_{q}=1-\sum_{i=1}^{q-1} X_{i}$.

Step 7: In case $X_{q}$ lies beyond the specified bounds, generate candidate design points. There may be multiple candidate points corresponding to a given design point. The candidate points are generated by adjusting the level of one of the components by a quantity equal to the difference between the substituted upper or lower bound and the computed value for $X_{q}$. Additional points are produced only from those components whose adjusted levels remain within the limits of the components.

We have illustrated the OABMD algorithm for generating mixture designs using orthogonal arrays based on difference schemes. These designs have been found to be efficient designs.

\section{Mixture Designs Developed using Difference Scheme}

4.1 Difference Scheme $D\left(p^{m}, p^{m}, p^{n}\right)$

A difference scheme $D\left(p^{m}, p^{m}, p^{n}\right)$ exists for any prime $p$ and integers $m \geq n \geq 1$ and consequently exists an $O A\left(p^{m} \times p^{n}, p^{m}, p^{n}, 2\right)$ and $O A\left(p^{m} \times p^{n}, p^{m}+1, p^{n}, 2\right)$.

\subsubsection{Example}

Consider a three-component mixture experiment, where all the components satisfy (1). Construct an orthogonal array $A=O A(9,3,3,2)$ with three factors, using the difference scheme $D(3,3,3)$.

$$
A^{T}=\left(\begin{array}{lllllllll}
0 & 0 & 0 & 1 & 1 & 1 & 2 & 2 & 2 \\
0 & 1 & 2 & 1 & 2 & 0 & 2 & 0 & 1 \\
0 & 2 & 1 & 1 & 0 & 2 & 2 & 1 & 0
\end{array}\right)
$$

Multiply it with a symmetric and non-orthogonal matrix, $M=p^{m} I-J$, where $I$ and $J$ denote identity matrix and a matrix of all ones respectively both of order $p^{m}$. In this case, $p^{m}=3$.

Multiply $A$ and $M$ to obtain $T$,

$$
T^{T}=\left(\begin{array}{lllllllll}
3 & 0 & 0 & 3 & 3 & 3 & 3 & 6 & 6 \\
3 & 3 & 6 & 3 & 6 & 0 & 3 & 0 & 3 \\
3 & 6 & 3 & 3 & 0 & 6 & 3 & 3 & 0
\end{array}\right)
$$


International Journal of Mathematical, Engineering and Management Sciences

Vol. 5, No. 6, 1379-1391, 2020

https://doi.org/10.33889/IJMEMS.2020.5.6.102

Using step 4 of the OABMD algorithm, we obtain the design matrix $\mathrm{Z}$ for unrestricted region as follows:

$$
Z^{T}=\left(\begin{array}{ccccccccc}
0.33 & 0 & 0 & 0.33 & 0.33 & 0.33 & 0.33 & 0.67 & 0.67 \\
0.33 & 0.33 & 0.67 & 0.33 & 0.67 & 0 & 0.33 & 0 & 0.33 \\
0.34 & 0.67 & 0.33 & 0.34 & 0 & 0.67 & 0.34 & 0.33 & 0
\end{array}\right)
$$

We observe that $0 \leq z_{i} \leq 1$ and sum of the elements for each row is unity. This design has a Gefficiency of $75 \%, 80 \%$ and $86.15 \%$ for fitting (3), (4) and (5) respectively. However, we observe that there are two repeat runs in $Z$. After the omission of the repeat runs from the unconstrained design matrix, $Z$ yields a G-efficiency of $90 \%, 85.71 \%$ and $76.59 \%$ for fitting (3), (4) and (5) respectively.

Consider the following three component mixture experiment (Snee and Marquardt, 1974; Saxena and Nigam, 1977), in order of increasing ranges:

$0.1 \leq X_{1} \leq 0.6,0.1 \leq X_{2} \leq 0.7$ and $0 \leq X_{3} \leq 0.7$

Using steps 5 and 6 of the OABMD algorithm, the constrained mixture design, $X$, is

$X^{T}=\left(\begin{array}{lllllllll}0.27 & 0.10 & 0.10 & 0.27 & 0.27 & 0.27 & 0.27 & 0.43 & 0.43 \\ 0.30 & 0.30 & 0.50 & 0.30 & 0.50 & 0.10 & 0.30 & 0.10 & 0.30 \\ 0.43 & 0.60 & 0.40 & 0.43 & 0.23 & 0.63 & 0.43 & 0.47 & 0.27\end{array}\right)$

This nine-run design matrix has a G-efficiency of 75\%,80\% and $77.77 \%$ for fitting (3), (4) and (5) respectively as compared to the nine-run design matrix composed by Saxena and Nigam (1977) yielding a G-efficiency of $68.25 \%, 74.70 \%$ and $78.66 \%$ for fitting (3), (4) and (5) respectively. Considering only the seven distinct runs of the design matrix $X$, gives a G-efficiency of $90 \%$, $85.71 \%$ and $100 \%$ for fitting (3), (4) and (5) respectively.

Other choices of $M$ with corresponding G-efficiency values of $Z$ and $X$ for fitting Scheffé's canonical polynomials are listed in Table 1 .

Table 1. G-efficiency of unconstrained and constrained mixture designs corresponding to different choices of $M$ for fitting Scheffe's canonical polynomials

\begin{tabular}{|c|c|c|c|c|c|}
\hline \multirow{2}{*}{\multicolumn{2}{|c|}{ Choice of $M$}} & \multirow{2}{*}{$\begin{array}{l}\text { G-efficiency for fitting } \\
\text { (3) for } Z \text { and } X\end{array}$} & \multirow{2}{*}{$\begin{array}{l}\text { G-efficiency for fitting (4) } \\
\text { for } Z \text { and } X\end{array}$} & \multicolumn{2}{|c|}{ G-efficiency for fitting (5) } \\
\hline & & & & $Z$ & $X$ \\
\hline$M_{1}=$ & $\left.\begin{array}{r}0 \\
-1 \\
1\end{array}\right)$ & $75 \%$ & $80 \%$ & $80.87 \%$ & $77.77 \%$ \\
\hline \multicolumn{2}{|c|}{$M_{2}=-M_{1}$} & $75 \%$ & $80 \%$ & $82.15 \%$ & $77.77 \%$ \\
\hline$M_{3}=$ & $\left.\begin{array}{r}0 \\
1 \\
-1\end{array}\right)$ & $75 \%$ & $80 \%$ & $77.77 \%$ & $77.77 \%$ \\
\hline \multicolumn{2}{|c|}{$M_{4}=-M_{3}$} & $75 \%$ & $80 \%$ & $77.77 \%$ & $77.77 \%$ \\
\hline \multicolumn{2}{|c|}{$M_{5}=-M$} & $75 \%$ & $80 \%$ & $70 \%{ }^{\#}$ & $77.77 \%$ \\
\hline
\end{tabular}

\#Obtained after addition of an extreme vertex, $(1,0,0)$, to the resulting 9-run design matrix, which initially was giving a G-efficiency of $27.63 \%$ for fitting (5). 
International Journal of Mathematical, Engineering and Management Sciences

Vol. 5, No. 6, 1379-1391, 2020

https://doi.org/10.33889/IJMEMS.2020.5.6.102

Remarkably, it is detected that the G-efficiency of the unconstrained mixture design constructed using the orthogonal array $A$ with any choice of $M$ (as listed in Table 1) is $75 \%$ for fitting Scheffé's linear model and $80 \%$ for fitting Scheffé's quadratic model. The same result holds for the constrained three component mixture experiment (Snee and Marquardt, 1974; Saxena and Nigam, 1977).

\subsubsection{Example}

Consider a four-component mixture experiment, where all the components satisfy (1). Construct an orthogonal array $A^{*}=O A(16,4,4,2)$ with four factors, using the difference scheme $D(4,4,4)$.

$$
A^{* T}=\left(\begin{array}{llllllllllllllll}
0 & 0 & 0 & 0 & 1 & 1 & 1 & 1 & 2 & 2 & 2 & 2 & 3 & 3 & 3 & 3 \\
0 & 1 & 2 & 3 & 1 & 0 & 3 & 2 & 2 & 3 & 0 & 1 & 3 & 2 & 1 & 0 \\
0 & 2 & 3 & 1 & 1 & 3 & 2 & 0 & 2 & 0 & 1 & 3 & 3 & 1 & 0 & 2 \\
0 & 3 & 1 & 2 & 1 & 2 & 0 & 3 & 2 & 1 & 3 & 0 & 3 & 0 & 2 & 1
\end{array}\right)
$$

Using a symmetric and non-orthogonal matrix, $M^{*}=p^{m} I-J$, where $I$ and $J$ denote identity matrix and a matrix of all ones respectively both of order $p^{m}$. In this case, $p^{m}=4$.

The unconstrained mixture design $Z^{*}$ is,

$$
Z^{* T}=\left(\begin{array}{cccccccccccccccc}
0.25 & 0 & 0 & 0 & 0.25 & 0.17 & 0.17 & 0.17 & 0.25 & 0.33 & 0.33 & 0.33 & 0.25 & 0.50 & 0.50 & 0.50 \\
0.25 & 0.17 & 0.33 & 0.50 & 0.25 & 0 & 0.50 & 0.33 & 0.25 & 0.50 & 0 & 0.17 & 0.25 & 0.33 & 0.17 & 0 \\
0.25 & 0.33 & 0.50 & 0.17 & 0.25 & 0.50 & 0.33 & 0 & 0.25 & 0 & 0.17 & 0.50 & 0.25 & 0.17 & 0 & 0.33 \\
0.25 & 0.50 & 0.17 & 0.33 & 0.25 & 0.33 & 0 & 0.50 & 0.25 & 0.17 & 0.50 & 0 & 0.25 & 0 & 0.33 & 0.17
\end{array}\right)
$$

This design matrix consists of three repeat runs and has a G-efficiency of $80 \%, 83.33 \%$ and $70.88 \%$ for fitting (3), (4) and (5) respectively.

Consider the constrained four component mixture experiment (McLean and Anderson, 1966). In manufacturing one particular type of flare the chemical constituents are magnesium $\left(x_{1}\right)$, sodium nitrate $\left(x_{2}\right)$, strontium nitrate $\left(x_{3}\right)$, and binder $\left(x_{4}\right)$. Engineering experience has indicated that the following constraints (in order of increasing ranges) on a proportion by weight basis should be utilized:

$$
0.03 \leq X_{1} \leq 0.08,0.40 \leq X_{2} \leq 0.60,0.10 \leq X_{3} \leq 0.50 \text { and } 0.10 \leq X_{4} \leq 0.50 \text {. }
$$

\begin{tabular}{|c|c|c|c|c|c|c|c|c|c|c|c|c|c|c|c|c|}
\hline \multirow{4}{*}{$X^{* T}=$} & 0.0425 & 0.03 & 0.03 & 0.03 & 0.0425 & 0.0383 & 0.0383 & 0.0383 & 0.0425 & 0.0467 & 0.0467 & 0.0467 & 0.0425 & 0.055 & 0.055 & 0.055 \\
\hline & 0.45 & 0.43 & 0.47 & 0.50 & 0.45 & 0.40 & 0.50 & 0.47 & 0.45 & 0.50 & 0.40 & 0.43 & 0.45 & 0.47 & 0.43 & 0.40 \\
\hline & 0.20 & 0.23 & 0.30 & 0.17 & 0.20 & 0.30 & 0.23 & 0.10 & 0.20 & 0.10 & 0.17 & 0.30 & 0.20 & 0.17 & 0.10 & 0.23 \\
\hline & \0.3075 & 0.31 & 0.20 & 0.30 & 0.3075 & 0.2617 & 0.2317 & 0.3917 & 0.3075 & 0.3533 & 0.3833 & 0.2233 & 0.3075 & 0.305 & 0.415 & 0.315 \\
\hline
\end{tabular}

Using the OABMD algorithm, the constrained mixture design, $X^{*}$, is

This design has thirteen distinct runs and a G-efficiency of $80 \%, 83.33 \%$ and $84.70 \%$ for fitting (3), (4) and (5) respectively as compared to the fifteen design runs considered by McLean and Anderson (1966) yielding a G-efficiency of 57.16\%, 71.24\% and 93.33\% for fitting (3), (4) and (5) respectively. Considering only the non-repeat runs in both the design matrices, $Z^{*}$ and $X^{*}$, yields a G-efficiency of $94.11 \%$ and $76.92 \%$ for fitting (3) and (4) respectively. Other choices of $M^{*}$ with corresponding G-efficiency values of $Z^{*}$ and $X^{*}$ for fitting Scheffé's canonical polynomials are listed in Table 2. 
International Journal of Mathematical, Engineering and Management Sciences

Vol. 5, No. 6, 1379-1391, 2020

https://doi.org/10.33889/IJMEMS.2020.5.6.102

Table 2. G-efficiency of unconstrained and constrained mixture designs corresponding to different choices of $M^{*}$ for fitting Scheffé's canonical polynomials

\begin{tabular}{|c|c|c|c|c|c|c|c|c|}
\hline \multirow{2}{*}{\multicolumn{5}{|c|}{ Choice of $M^{*}$}} & \multirow{2}{*}{$\begin{array}{l}\text { G-efficiency for fitting } \\
\text { (3) for } Z^{*} \text { and } X^{*}\end{array}$} & \multirow{2}{*}{$\begin{array}{l}\text { G-efficiency for fitting } \\
\text { (4) for } Z^{*} \text { and } X^{*}\end{array}$} & \multicolumn{2}{|c|}{ G-efficiency for fitting (5) } \\
\hline & & & & & & & $\boldsymbol{Z}^{*}$ & $\boldsymbol{X}^{*}$ \\
\hline$M_{1}^{*}=$ & $\begin{array}{r}1 \\
-1 \\
0 \\
0\end{array}$ & $\begin{array}{r}-1 \\
2 \\
-1 \\
0\end{array}$ & $\begin{array}{r}0 \\
-1 \\
2 \\
-1\end{array}$ & $\left.\begin{array}{r}0 \\
0 \\
-1 \\
1\end{array}\right)$ & $80 \%$ & $83.33 \%$ & $79.60 \%$ & $86.67 \%$ \\
\hline \multicolumn{5}{|c|}{$M_{2}^{*}=-M_{1}^{*}$} & $80 \%$ & $83.33 \%$ & $79.60 \%$ & $80.89 \%$ \\
\hline$M_{3}^{*}=$ & $\left(\begin{array}{r}1 \\
-1 \\
0 \\
0\end{array}\right.$ & $\begin{array}{r}-1 \\
0 \\
1\end{array}$ & $\begin{array}{r}0 \\
1 \\
0 \\
-1\end{array}$ & $\left.\begin{array}{r}0 \\
0 \\
-1 \\
1\end{array}\right)$ & $80 \%$ & $83.33 \%$ & $60.41 \%$ & $59.95 \%$ \\
\hline \multicolumn{5}{|c|}{$M_{4}^{*}=M_{3}^{*}$} & $80 \%$ & $83.33 \%$ & $69.35 \%$ & $78.99 \%$ \\
\hline \multicolumn{5}{|c|}{$M_{5}^{*}=-M^{*}$} & $80 \%$ & $83.33 \%$ & $85.64 \%$ & $85.04 \%$ \\
\hline
\end{tabular}

Interestingly, it is observed that the G-efficiency of the unconstrained mixture design constructed using the orthogonal array $A^{*}$ with any choice of $M^{*}$ (as listed in Table 2) is $80 \%$ for fitting Scheffé's linear model and $83.33 \%$ for fitting Scheffé's quadratic model. The same result holds for the constrained four component mixture experiment (McLean and Anderson, 1966).

\subsubsection{Example}

A difference scheme $D(p, p, p)$ can be used to construct an orthogonal array $O A\left(p^{2}, p+1, p, 2\right)$ of index unity.

Construct an orthogonal array $B=O A(16,5,4,2)$ in five factors, by appending a symbol to each of the runs in $A^{*}=O A(16,4,4,2)$.

$B^{T}=\left(\begin{array}{llllllllllllllll}0 & 0 & 0 & 0 & 1 & 1 & 1 & 1 & 2 & 2 & 2 & 2 & 3 & 3 & 3 & 3 \\ 0 & 1 & 2 & 3 & 1 & 0 & 3 & 2 & 2 & 3 & 0 & 1 & 3 & 2 & 1 & 0 \\ 0 & 2 & 3 & 1 & 1 & 3 & 2 & 0 & 2 & 0 & 1 & 3 & 3 & 1 & 0 & 2 \\ 0 & 3 & 1 & 2 & 1 & 2 & 0 & 3 & 2 & 1 & 3 & 0 & 3 & 0 & 2 & 1 \\ 0 & 1 & 2 & 3 & 0 & 1 & 2 & 3 & 0 & 1 & 2 & 3 & 0 & 1 & 2 & 3\end{array}\right)$

Using a symmetric and non-orthogonal matrix, $M^{* *}=(p+1) I-J$, where $I$ and $J$ denote identity matrix and a matrix of all ones respectively both of order $p+1$. In this case, $p=4$.

The unconstrained mixture design $Z_{1}$ is,

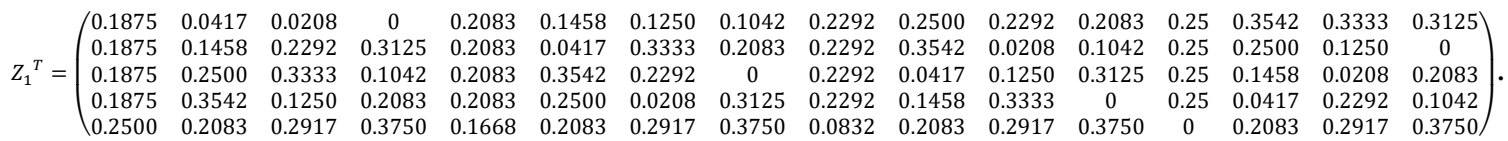

This design matrix consists of all distinct runs and has a G-efficiency of $73.96 \%$ and $93.75 \%$ for fitting (3) and (4) respectively. 
International Journal of Mathematical, Engineering and Management Sciences

Vol. 5, No. 6, 1379-1391, 2020

https://doi.org/10.33889/IJMEMS.2020.5.6.102

Consider the lubricating oil example with five-components (Saxena and Nigam, 1977). In manufacturing a specific type of lubricating oil for the internal combustion engine, the constituents are Pour Point Depressant $\left(x_{1}\right)$, Detergent Dispersant $\left(x_{2}\right)$, Anti-Oxidants $\left(x_{3}\right)$, Viscosity Index Improvers $\left(x_{4}\right)$ and the base, SAE-10 oil $\left(x_{5}\right)$. Experience has indicated that the following constraints on their proportions should be imposed: - Pour Point Depressant: $0.10 \%-0.50 \%$, Detergent Dispersant: 0.05\% - 1.00\%, Anti-Oxidants: 0.05\% - 1.00\%, Viscosity Index Improvers: $4.00 \%-10.00 \%$ and SAE-10 Oil: the remaining balance. The components ranked in the order of their ranges are $0.0010 \leq x_{1} \leq 0.0050,0.0005 \leq x_{2} \leq 0.0100,0.0005 \leq x_{3} \leq 0.0100$, $0.0400 \leq x_{4} \leq 0.1000$ and $0.8750 \leq x_{5} \leq 0.9580$.

Using the OABMD algorithm, the design matrix, $X_{1}$ is as follows:

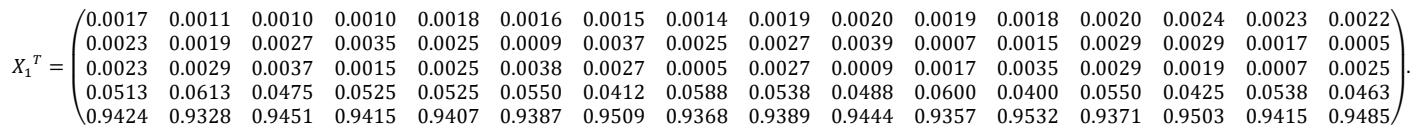

This design matrix also consists of all distinct runs and has a G-efficiency of $73.96 \%$ and $93.75 \%$ for fitting (3) and (4) respectively. On the contrary, the design matrix suggested by Saxena and Nigam (1977) had twenty design points having a G-efficiency of $49.78 \%$ and $76.62 \%$ for fitting (3) and (4) respectively. Other choices of $M^{* *}$ with corresponding G-efficiency values of unconstrained and constrained mixture design for fitting Scheffé's canonical polynomials are listed in Table 3.

Table 3. G-efficiency of unconstrained and constrained mixture designs corresponding to different choices of $M^{* *}$ for fitting Scheffé's canonical polynomials

\begin{tabular}{|c|c|c|c|c|c|}
\hline \multicolumn{4}{|c|}{ Choice of $M^{* *}$} & \multirow{2}{*}{$\begin{array}{c}\text { G-efficiency for fitting (3) for } \\
\boldsymbol{Z}_{\mathbf{1}} \text { and } \boldsymbol{X}_{\mathbf{1}} \\
\\
\\
73.96 \%\end{array}$} & \multirow{2}{*}{$\begin{array}{c}\text { G-efficiency for fitting (4) for } \\
\qquad \boldsymbol{Z}_{\mathbf{1}} \text { and } \boldsymbol{X}_{\mathbf{1}} \\
\\
93.75 \%\end{array}$} \\
\hline$M_{1}{ }^{* *}=$ & $\begin{array}{rr}1 & -1 \\
-1 & 2 \\
0 & -1 \\
0 & 0 \\
0 & 0\end{array}$ & $\begin{array}{rr}0 & 0 \\
-1 & 0 \\
2 & -1 \\
-1 & 2 \\
0 & -1\end{array}$ & $\left.\begin{array}{r}0 \\
0 \\
0 \\
-1 \\
1\end{array}\right)$ & & \\
\hline \multicolumn{4}{|c|}{$M_{2}{ }^{* *}=-M_{1}{ }^{* *}$} & $73.96 \%$ & $93.75 \%$ \\
\hline$M_{3}{ }^{* *}=$ & $\begin{array}{rr}1 & -1 \\
-1 & 0 \\
0 & 1 \\
0 & 0 \\
0 & 0\end{array}$ & $\begin{array}{rr}0 & 0 \\
1 & 0 \\
0 & -1 \\
-1 & 0 \\
0 & 1\end{array}$ & $\left.\begin{array}{r}0 \\
0 \\
0 \\
1 \\
-1\end{array}\right)$ & $73.96 \%$ & $93.75 \%$ \\
\hline \multicolumn{4}{|c|}{$M_{4}^{* *}=-M_{3}^{* *}$} & $73.96 \%$ & $93.75 \%$ \\
\hline$M_{5}^{* *}=$ & $\begin{array}{rr}0 & 1 \\
1 & 0 \\
-1 & 1 \\
-1 & -1 \\
1 & -1\end{array}$ & $\begin{array}{rr}-1 & -1 \\
1 & -1 \\
0 & 1 \\
1 & 0 \\
-1 & 1\end{array}$ & $\left.\begin{array}{r}1 \\
-1 \\
-1 \\
1 \\
0\end{array}\right)$ & $73.96 \%$ & $93.75 \%$ \\
\hline \multicolumn{4}{|c|}{$M_{6}{ }^{* *}=-M_{5}{ }^{* *}$} & $73.96 \%$ & $93.75 \%$ \\
\hline \multicolumn{4}{|c|}{$M_{7}^{* *}=-M^{* *}$} & $73.96 \%$ & $93.75 \%$ \\
\hline
\end{tabular}


Remarkably, it is detected that the G-efficiency of the unconstrained and constrained mixture design, the set of constraints as given by Saxena and Nigam (1977), constructed using any choice of $M^{* *}$ (as listed in Table 3) is $73.96 \%$ for fitting (3) and $93.75 \%$ for fitting (4).

\subsection{Difference Scheme $D(2 p, 2 p, p)$}

If $p$ is a power of an odd prime then there exists a difference scheme $D(2 p, 2 p, p)$ and an orthogonal array $O A\left(2 p^{2}, 2 p+1, p, 2\right)$.

\subsubsection{Example}

Construct an orthogonal array $C=O A(18,7,3,2)$ with seven factors, using the difference scheme $D(6,6,3)$.

$$
C^{T}=\left(\begin{array}{llllllllllllllllll}
0 & 0 & 0 & 0 & 0 & 0 & 1 & 1 & 1 & 1 & 1 & 1 & 2 & 2 & 2 & 2 & 2 & 2 \\
0 & 1 & 2 & 2 & 0 & 1 & 1 & 2 & 0 & 0 & 1 & 2 & 2 & 0 & 1 & 1 & 2 & 0 \\
0 & 2 & 1 & 2 & 1 & 0 & 1 & 0 & 2 & 0 & 2 & 1 & 2 & 1 & 0 & 1 & 0 & 2 \\
0 & 1 & 1 & 0 & 2 & 2 & 1 & 2 & 2 & 1 & 0 & 0 & 2 & 0 & 0 & 2 & 1 & 1 \\
0 & 2 & 0 & 1 & 2 & 1 & 1 & 0 & 1 & 2 & 0 & 2 & 2 & 1 & 2 & 0 & 1 & 0 \\
0 & 0 & 2 & 1 & 1 & 2 & 1 & 1 & 0 & 2 & 2 & 0 & 2 & 2 & 1 & 0 & 0 & 1 \\
0 & 0 & 1 & 1 & 2 & 2 & 0 & 0 & 1 & 1 & 2 & 2 & 0 & 0 & 1 & 1 & 2 & 2
\end{array}\right)
$$

Using a symmetric and non-orthogonal matrix, $M^{* * *}=(2 p+1) I-J$, where $I$ and $J$ denote identity matrix and a matrix of all ones respectively both of order $(2 p+1)$. In this case, $p=3$.

The unconstrained mixture design $Z_{2}$ is,

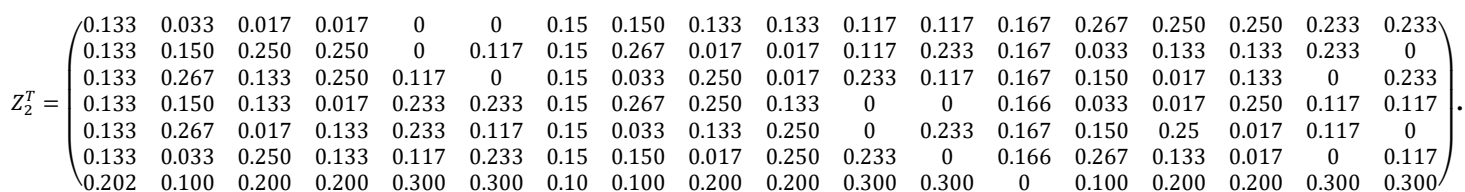

This design matrix consists of all distinct runs and has a G-efficiency of $84.48 \%$ for fitting Scheffé's linear model.

Other choices of $M^{* * *}$ with corresponding G-efficiency values of unconstrained mixture design for fitting Scheffé's linear model are listed in Table 4. 
International Journal of Mathematical, Engineering and Management Sciences

Vol. 5, No. 6, 1379-1391, 2020

https://doi.org/10.33889/IJMEMS.2020.5.6.102

Table 4. G-efficiency of unconstrained mixture designs corresponding to different choices of $M^{* * *}$ for fitting Scheffé's linear model

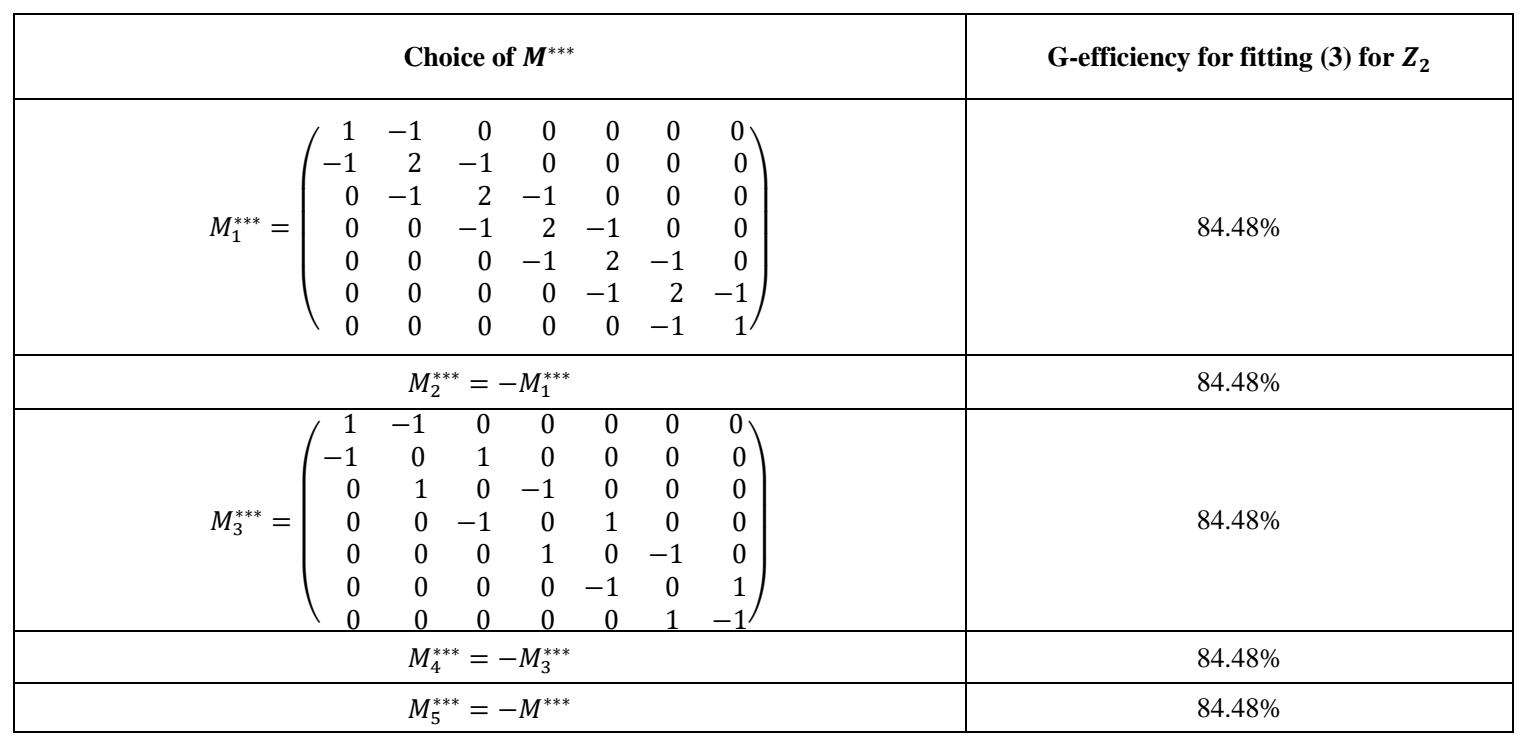

It can be observed from the above table that the G-efficiency for fitting (3) is $84.48 \%$ for the unconstrained design matrix attained by using the orthogonal array $C$ with any choice of the $M^{* * *}$ matrix (as listed in Table 4). However, in case fitting of higher order models (4) and (5) is required, the boundary points, mid-point of the design runs or the extreme vertices may be added to the existing design matrix for fitting the models and subsequently the computation of G-efficiency.

\subsection{Difference Scheme $D_{t}(r, q, s)$ of Strength $t$}

A difference scheme $D_{t}(r, q, s)$ of strength $t$ can be used to construct an $O A(r s, q, s, t)$. If the difference scheme itself is already an orthogonal array of strength $t-1$ or if it can be written as the juxtaposition of $s$ difference schemes $D_{t-1}(r / s, q, s)$, then it can be used to construct an $O A(r s, q+1, s, t)$.

\subsubsection{Example}

Construct an orthogonal array $E=O A(16,8,2,3)$ with eight factors, using the difference scheme $D_{3}(8,7,2)$.

$$
E^{T}=\left(\begin{array}{llllllllllllllll}
0 & 0 & 0 & 0 & 1 & 1 & 1 & 1 & 1 & 1 & 1 & 1 & 0 & 0 & 0 & 0 \\
0 & 0 & 1 & 1 & 0 & 0 & 1 & 1 & 1 & 1 & 0 & 0 & 1 & 1 & 0 & 0 \\
0 & 1 & 0 & 1 & 0 & 1 & 0 & 1 & 1 & 0 & 1 & 0 & 1 & 0 & 1 & 0 \\
0 & 0 & 1 & 1 & 1 & 1 & 0 & 0 & 1 & 1 & 0 & 0 & 0 & 0 & 1 & 1 \\
0 & 1 & 0 & 1 & 1 & 0 & 1 & 0 & 1 & 0 & 1 & 0 & 0 & 1 & 0 & 1 \\
0 & 1 & 1 & 0 & 0 & 1 & 1 & 0 & 1 & 0 & 0 & 1 & 1 & 0 & 0 & 1 \\
0 & 1 & 1 & 0 & 1 & 0 & 0 & 1 & 1 & 0 & 0 & 1 & 0 & 1 & 1 & 0 \\
0 & 0 & 0 & 0 & 0 & 0 & 0 & 0 & 1 & 1 & 1 & 1 & 1 & 1 & 1 & 1
\end{array}\right) .
$$

Using a symmetric and non-orthogonal matrix, $M^{* * * *}=(q+1) I-J$, where $I$ and $J$ denote identity matrix and a matrix of all ones respectively both of order $(q+1)$. In this case, $q=7$. 
International Journal of Mathematical, Engineering and Management Sciences

Vol. 5, No. 6, 1379-1391, 2020

https://doi.org/10.33889/IJMEMS.2020.5.6.102

The unconstrained mixture design $Z_{3}$ is,

$Z_{3}^{T}=\left(\begin{array}{cccccccccccccccc}0.125 & 0 & 0 & 0 & 0.25 & 0.25 & 0.25 & 0.25 & 0.125 & 0.25 & 0.25 & 0.25 & 0 & 0 & 0 & 0 \\ 0.125 & 0 & 0.25 & 0.25 & 0 & 0 & 0.25 & 0.25 & 0.125 & 0.25 & 0 & 0 & 0.25 & 0.25 & 0 & 0 \\ 0.125 & 0.25 & 0 & 0.25 & 0 & 0.25 & 0 & 0.25 & 0.125 & 0 & 0.25 & 0 & 0.25 & 0 & 0.25 & 0 \\ 0.125 & 0 & 0.25 & 0.25 & 0.25 & 0.25 & 0 & 0 & 0.125 & 0.25 & 0 & 0 & 0 & 0 & 0.25 & 0.25 \\ 0.125 & 0.25 & 0 & 0.25 & 0.25 & 0 & 0.25 & 0 & 0.125 & 0 & 0.25 & 0 & 0 & 0.25 & 0 & 0.25 \\ 0.125 & 0.25 & 0.25 & 0 & 0 & 0.25 & 0.25 & 0 & 0.125 & 0 & 0 & 0.25 & 0.25 & 0 & 0 & 0.25 \\ 0.125 & 0.25 & 0.25 & 0 & 0.25 & 0 & 0 & 0.25 & 0.125 & 0 & 0 & 0.25 & 0 & 0.25 & 0.25 & 0 \\ 0.125 & 0 & 0 & 0 & 0 & 0 & 0 & 0 & 0.125 & 0.25 & 0.25 & 0.25 & 0.25 & 0.25 & 0.25 & 0.25\end{array}\right)$.

This design matrix consists a repeat run and has a G-efficiency of $88.88 \%$ for fitting Scheffé's linear model. Nevertheless, considering only the distinct runs of the design matrix $Z_{3}$ yields a Gefficiency of $94.11 \%$ for fitting (3).

Other choices of $M^{* * * *}$ with corresponding G-efficiency values of unconstrained mixture design for fitting Scheffé's linear model are listed in Table 5.

Table 5. G-efficiency of unconstrained mixture designs corresponding to different choices of $M^{* * * *}$ for fitting Scheffé's linear model

\begin{tabular}{|c|c|c|c|c|c|c|c|c|c|c|}
\hline \multicolumn{9}{|c|}{ Choice of $\boldsymbol{M}^{* * * *}$} & $\begin{array}{c}\text { G-efficiency for fitting (3) } \\
\text { for } Z_{3}\end{array}$ & $\begin{array}{c}\text { G-efficiency for fitting (3) } \\
\text { for } Z_{3} \text { after omitting the } \\
\text { repeat run }\end{array}$ \\
\hline$M_{1}^{* * * *}=$ & $\begin{array}{r}1 \\
-1 \\
0 \\
0 \\
0 \\
0 \\
0 \\
0 \\
\end{array}$ & $\begin{array}{r}-1 \\
2 \\
-1 \\
0 \\
0 \\
0 \\
0 \\
0 \\
\end{array}$ & $\begin{array}{r}0 \\
-1 \\
2 \\
-1 \\
0 \\
0 \\
0 \\
0 \\
\end{array}$ & $\begin{array}{r}0 \\
0 \\
-1 \\
2 \\
-1 \\
0 \\
0 \\
0 \\
\end{array}$ & $\begin{array}{r}0 \\
0 \\
0 \\
-1 \\
2 \\
-1 \\
0 \\
0 \\
\end{array}$ & $\begin{array}{r}0 \\
0 \\
0 \\
0 \\
-1 \\
2 \\
-1 \\
0 \\
\end{array}$ & $\begin{array}{r}0 \\
0 \\
0 \\
0 \\
0 \\
-1 \\
2 \\
-1 \\
\end{array}$ & $\left.\begin{array}{r}0 \\
0 \\
0 \\
0 \\
0 \\
0 \\
-1 \\
1\end{array}\right)$ & $88.88 \%$ & $94.11 \%$ \\
\hline \multicolumn{9}{|c|}{$M_{2}^{* * * *}=-M_{1}^{* * * *}$} & $88.88 \%$ & $94.11 \%$ \\
\hline$M_{3}{ }^{* * * *}=$ & $\left(\begin{array}{r}1 \\
-1 \\
0 \\
0 \\
0 \\
0 \\
0 \\
0\end{array}\right.$ & $\begin{array}{r}-1 \\
0 \\
1 \\
0 \\
0 \\
0 \\
0 \\
0\end{array}$ & $\begin{array}{r}0 \\
1 \\
0 \\
-1 \\
0 \\
0 \\
0 \\
0\end{array}$ & $\begin{array}{r}0 \\
0 \\
-1 \\
0 \\
1 \\
0 \\
0 \\
0\end{array}$ & $\begin{array}{r}0 \\
0 \\
0 \\
1 \\
0 \\
-1 \\
0 \\
0\end{array}$ & $\begin{array}{r}0 \\
0 \\
0 \\
0 \\
-1 \\
0 \\
1 \\
0\end{array}$ & $\begin{array}{r}0 \\
0 \\
0 \\
0 \\
0 \\
1 \\
0 \\
-1\end{array}$ & $\left.\begin{array}{r}0 \\
0 \\
0 \\
0 \\
0 \\
0 \\
-1 \\
1\end{array}\right)$ & $88.88 \%$ & $94.11 \%$ \\
\hline \multicolumn{9}{|c|}{$M_{4}^{* * * *}=-M_{3}^{* * * *}$} & $88.88 \%$ & $94.11 \%$ \\
\hline \multicolumn{9}{|c|}{$M_{5}^{* * * *}=-M^{* * * *}$} & $88.88 \%$ & $94.11 \%$ \\
\hline
\end{tabular}

Interestingly, the same value of G-efficiency for fitting (3) is achieved for the unconstrained design matrix obtained by using the orthogonal array $E$ with any choice of the $M^{* * * *}$ matrix (as listed in Table 5). However, in case fitting of higher order models (4) and (5) is required, the boundary points, mid-point of the design runs or the extreme vertices may be added to the existing design matrix for fitting the models and subsequently the computation of G-efficiency.

\section{Conclusion}

The orthogonal arrays developed using difference schemes have been considered in our proposed OABMD algorithm. The designs, hence, constructed have manageable number of design points that help in reducing the cost and time in statistical experiments. Furthermore, the flexibility in 
International Journal of Mathematical, Engineering and Management Sciences

Vol. 5, No. 6, 1379-1391, 2020

https://doi.org/10.33889/IJMEMS.2020.5.6.102

choice of matrix $M$ allows for enhanced variety of design points. When the region of interest is predefined, the proposed OABMD algorithm can be customized to explore a restricted space.

The constructed designs have a sufficiently high G-efficiency that make them suitable for practical purposes.

Conflict of Interest

The authors declare that there is no conflict of interest for this publication.

\section{Acknowledgment}

This research did not receive funding from any public, commercial or not-for-profit sector. The authors are thankful to the editor and the referees for their valuable suggestions that helped in the improvement of the paper.

\section{References}

Bose, R.C., \& Bush, K.A. (1952). Orthogonal arrays of strength two and three. The Annals of Mathematical Statistics, 23, 508-524.

Bush, K.A. (1952). Orthogonal arrays of index unity. The Annals of Mathematical Statistics 23, 426-434.

Jungnickel, D. (1979). On difference matrices, resolvable transversal designs and generalized Hadamard matrices. Mathematische Zeitschrift, 167(1), 49-60.

Masuyama, M. (1969). Cyclic generation of $O A\left(2 s^{m}, 2\left(s^{m}-1\right) /(s-1)-1, s, 2\right)$. Rep. Statist. Appl. Res., JUSE, 16, 10-16.

McLean, R.A., \& Anderson, V.L. (1966). Extreme vertices design of mixture experiments. Technometrics, $8(3), 447454$.

Mukhopadhyay, A.C. (1981). Construction of some series of orthogonal arrays. Sankhya: The Indian Journal of Statistics, Series B, 43(1), 81-92.

Murthy, M.S.R., \& Murty, J.S. (1983). Restricted region simplex design for mixture experiments. Communication in Statistics Theory and Methods, 12(22), 2605-2615.

Nigam, A.K., Gupta, S.C., \& Gupta, S. (1983). An algorithm for extreme vertices designs for linear mixture models. Technometrics, 25(4), 367-371.

Rao, C.R. (1946). Hypercubes of strength " $d$ " leading to confounded designs in factorial experiments. Bulletin of the Calcutta Mathematical Society, 38, 67-78.

Rao, C.R. (1947). Factorial experiments derivable from combinatorial arrangements of arrays. Supplement to the Journal of Royal Statistical Society, 9(1), 128-139.

Saxena, S.K., \& Nigam, A.K. (1977). Restricted exploration of mixtures by symmetric simplex design. Technometrics, 19(1), 47-52.

Scheffé, H. (1958). Experiments with mixtures. Journal of the Royal Statistical Society. Series B (Methodological), 20(2), 344-360.

Scheffé, H. (1963). The simplex centroid design for experiments with mixtures. Journal of the Royal Statistical Society, Series B (Methodological), 25(2), 235-251. 
International Journal of Mathematical, Engineering and Management Sciences

Vol. 5, No. 6, 1379-1391, 2020

https://doi.org/10.33889/IJMEMS.2020.5.6.102

Shrikhande, S.S. (1964). Generalized hadamard matrices and orthogonal arrays of strength two. Canadian Journal of Mathematics, 16, 736-740.

Singh, P., Sarin, V., \& Midha, N. (2018). Construction of mixture designs based on taguchi's fixed element orthogonal arrays. International Journal of Statistics and Reliability Engineering, 5(2), 89-95.

Snee, R.D. (1975). Experimental designs for quadratic models in constrained mixture spaces. Technometrics, 17(2), 149-159.

Snee, R.D., \& Marquardt, D.W. (1974). Extreme vertices designs for linear mixture models. Technometrics, 16(3), 399-408.

Thompson, W.O., \& Myers, R.H. (1968). Response surface designs for experiments with mixtures. Technometrics, 10(4), 739-756.

Wheeler, R.E. (1972). Efficient experimental design. Joint statistical meetings of the American Statistical Association, IMS and Biometric Society, Montreal, Canada.

Xiang, K.F. (1983). The difference set table for $\lambda=2$. (Chinese). Acta Mathematicae Applicatae Sinica, 6(2), 160-166.

Xu, C.X. (1979). Construction of orthogonal arrays $L_{2} p^{u}\left(p^{1+\sum_{i=1}^{u-1} 2 p^{i}}\right)$ with odd prime $p$. (Chinese). Acta Mathematicae Applicatae Sinica, 2, 92-97. 